Revista Electrónica Complutense de Investigación en Educación Musical ISSNe: 1698-7454

http://dx.doi.org/10.5209/RECIEM.54844

\title{
La Educación Musical en el actual currículo español. ¿Qué formación recibe el alumnado en la enseñanza Primaria?
}

\author{
Oscar Casanova $^{1}$; Rosa María Serrano ${ }^{2}$
}

Recibido: 30 de diciembre 2016 / Aceptado: 21 de enero 2018

Resumen. La legislación educativa, tanto estatal como sus concreciones autonómicas, establecen el marco sobre el que se asienta la Educación Musical en la etapa de Primaria. Este documento estudia en profundidad, desde una perspectiva cualitativa, la normativa actual de las diferentes Administraciones con el fin de comprender la posición que ocupa la enseñanza musical a lo largo del territorio español y conocer cuál es el aprendizaje buscado en el alumnado, comprobando si existen diferencias educativas significativas dependiendo del lugar en el que se viva. Se analizan los elementos que constituyen el currículo, prestando especial atención a la dedicación temporal y los aprendizajes musicales pretendidos. Tras la revisión bibliográfica de estos textos legislativos, se apuntan las posibles repercusiones para el futuro de la Educación Musical obligatoria para esta etapa.

Palabras clave: Educación Musical; Educación Primaria; currículo; legislación educativa.

\section{[en] The Music Education in the current Spanish curriculum. What training does the pupils receive in Primary education?}

\begin{abstract}
Educational legislation, both state and its regional concretions, establishes the framework of Music Education in Primary school. This document studies in depth, from a qualitative perspective, the current regulations of the different Administrations in order to understand the position that music teaching occupies throughout Spanish territory and to know what students are learning, checking if there are significant educational differences depending on the place where they live. The components of the curriculum are analysed, paying special attention to temporal dedication and musical learning. The bibliographic revision of these legislative texts, points out the possible repercussions for the future of compulsory music education in Primary school.
\end{abstract}

Keywords: Music education; Primary education; curriculum; educational legislation.

Sumario. 1. Introducción. 2. El marco legislativo estatal. 3. Metodología. 4. Descripción e interpretación de los datos. 5. Discusión y conclusiones. 6. Repercusiones y prospectiva. 7. Referencias bibliográficas.

Cómo citar. Casanova, O. y Serrano, R. M. (2018). La educación musical en el actual currículo español. ¿Qué formación recibe el alumnado en la enseñanza Primaria? Revista Electrónica Complutense de Investigación en Educación Musical, 15, 3-17.

\footnotetext{
1 Facultad de Educación. Universidad de Zaragoza (España). ocasanov@unizar.es

2 Facultad de Educación. Universidad de Zaragoza (España). rmserran@unizar.es
} 


\section{Introducción}

La educación en España está en unos momentos de transformación y renovación pedagógica. "La situación económica y social actual, junto con los retos que la sociedad contemporánea presenta, han llevado a las autoridades políticas y educativas a apostar por la reformulación de la existente ley educativa" (Cabedo y Díaz, 2015, p. 271), así como a realizar las modificaciones que de manera paralela obliga. Tal y como indica Aróstegui (2014b) la manera en la que se enfoca el propósito y el modo de la educación en cada una estas situaciones socio-económicas es lo que denominamos currículo, a través del cual se defienden teorías educativas distintas que ponen el énfasis en aspectos diferentes de lo que debe ser la enseñanza escolar, porque, evidentemente, la educación es una actividad ideológica y moral.

La normativa actual se fundamenta en un determinado desarrollo competencial (Rodríguez y González, 2013) y evaluación, así como en otros aspectos como la valoración de ciertas asignaturas que considera troncales. Dicha normativa ha sido aprobada pese a una gran oposición política, social y ciudadana (Rodríguez, 2014). Sea como fuere, este enfoque particular repercute de manera directa en el lugar que permiten ocupar a la Educación Musical en la actualidad.

Un modelo educativo, aunque descentralizado, no debería tener grandes diferencias en su aplicación respecto a la educación obligatoria. Es por ello que se hace necesario un estudio y análisis profundo de la concreción curricular efectuada por cada Comunidad Autónoma que permita conocer con exactitud y reflexionar sobre las posibles repercusiones para el futuro de la Educación Musical obligatoria para esta etapa.

\section{EI marco legislativo estatal}

Es necesario detallar la actual legislación estatal en Educación Primaria como paso previo a la revisión bibliográfica de los correspondientes textos legislativos autonómicos y su posterior análisis comparativo. El fin es ofrecer un punto de partida sobre la Legislación Educativa que en este documento se analiza, presentando a continuación, de manera general y descriptiva, las características esenciales de la normativa estatal que es objeto de concreción curricular por las diferentes Administraciones educativas.

El Real Decreto 126/2014, de 28 de febrero, por el que se establece el currículo básico de la Educación Primaria, implanta en España la nueva organización de esta etapa educativa que se desarrolla en los artículos 16 a 21 de la Ley Orgánica 2/2006, de 3 de mayo, de Educación (LOE) tras la modificación realizada por la Ley Orgánica 8/2013, de 9 de diciembre, para la Mejora de la Calidad Educativa (LOM$\mathrm{CE})$. Esta nueva Ley también modifica el artículo 6 de la LOE concretando que el currículo estará integrado por los objetivos de cada enseñanza y etapa educativa; las competencias, entendidas como aquellas capacidades para activar y aplicar de forma integrada los contenidos para lograr la realización adecuada de actividades y la resolución eficaz de problemas complejos; los contenidos, o conjuntos de conocimientos, habilidades, destrezas y actitudes que contribuyen al logro de los objetivos y a la adquisición de las competencias; la metodología didáctica, que comprende tanto la descripción de las prácticas docentes como la organización del trabajo docente; los 
estándares y resultados de aprendizaje evaluables; y los criterios de evaluación del grado de adquisición de las competencias y del logro de los objetivos. Asimismo, establece que los contenidos se ordenan en asignaturas, que se clasifican en materias, ámbitos, áreas y módulos en función de las enseñanzas o las etapas educativas.

En el incorporado artículo 6 bis de la LOMCE se especifican las competencias y funciones que le corresponde al Gobierno, al Ministerio de Educación, Cultura y Deporte, a las Administraciones educativas o Comunidades Autónomas, y a los centros educativos. Este mismo artículo indica que en Educación Primaria, en Educación Secundaria Obligatoria y en Bachillerato, las asignaturas se agruparán en tres bloques, de asignaturas troncales, de asignaturas específicas y de asignaturas de libre configuración autonómica; también se establece que el horario mínimo correspondiente al bloque de las asignaturas troncales, en cómputo global para toda la Educción Primaria, no será inferior al 50\% del total del horario lectivo establecido por cada una de las Administraciones educativas.

Sobre estas bases establecidas y competencias asignadas a cada uno de los estamentos, el Real Decreto 126/2014 regula el currículo básico de las diferentes áreas, organizándolo a partir de los objetivos propios de la etapa y de las siete competencias que se van a desarrollar. Establece los bloques de contenidos en las áreas troncales, y criterios de evaluación y estándares de aprendizaje evaluables en todas las áreas, para ser así referentes en la planificación de la concreción curricular y en la programación docente. Estos bloques permiten identificar los principales ámbitos que comprende el área, aunque, como también indica el propio Real Decreto, no implica una organización cerrada, permitiéndose organizar de diferentes maneras los elementos curriculares y adoptar la metodología más adecuada a las características de los mismos y del grupo de alumnos.

La mayor novedad del Real Decreto 126/2014 consiste en establecer unos estándares de aprendizaje evaluables, que define como:

Especificaciones de los criterios de evaluación que permiten definir los resultados de aprendizaje, y que concretan lo que el alumno debe saber, comprender y saber hacer en cada asignatura; deben ser observables, medibles y evaluables y permitir graduar el rendimiento o logro alcanzado. Su diseño debe contribuir y facilitar el diseño de pruebas estandarizadas y comparables. (pp. 19351-2)

Aclara también que los criterios de evaluación "son el referente específico para evaluar el aprendizaje del alumnado. Describen aquello que se quiere valorar y que el alumnado debe lograr, tanto en conocimientos como en competencias; responden a lo que se pretende conseguir en cada asignatura" (p. 19352).

También indica que la Educación Artística, área que la forman la música y la plástica, es colocada, y por tanto considerada, en el bloque de asignaturas específicas, y además en el grupo de las de posible optatividad, dejando la regulación y programación de su oferta a cada Administración educativa y, en su caso, los centros docentes.

En el artículo 8 se concretan todas las áreas que el alumnado deberá cursar, y la Educación Artística aparece en el apartado c), indicando que se cursará:

En función de la regulación y de la programación de la oferta educativa que establezca cada Administración educativa y, en su caso, de la oferta de los cen- 
tros docentes, al menos una de las siguientes áreas del bloque de asignaturas específicas:

$1^{\circ}$ Educación Artística.

$2^{\circ}$ Segunda Lengua Extranjera.

$3^{\circ}$ Religión, sólo si los padres, madres o tutores legales no la han escogido en la elección indicada en el apartado 3.b).

$4^{\circ}$ Valores Sociales y Cívicos, sólo si los padres, madres o tutores legales no la han escogido en la elección indicada en el apartado 3.b). (pp. 19354-5)

Además, se podrá cursar también:

[...] una o varias áreas más en el bloque de asignaturas de libre configuración autonómica, en función de la regulación y de la programación de la oferta educativa que establezca cada Administración educativa y, en su caso, de la oferta de los centros docentes, que podrán ser áreas del bloque de asignaturas específicas no cursadas, áreas de profundización o refuerzo de las áreas troncales, u otras áreas a determinar. (p. 19355)

Asimismo, el correspondiente anexo de asignaturas específicas establece que el área de Educación Artística se ha dividido en dos partes: la Educación Plástica y la Educación Musical, estableciendo para cada una de ellas tres bloques de contenidos. De los correspondientes a la música, el primero, escucha, es en el que el alumnado indagará en las posibilidades del sonido; el segundo bloque, la interpretación musical, comprende el desarrollo de habilidades para la interpretación musical; el tercer bloque, la música, el movimiento y la danza, es el destinado al desarrollo de capacidades expresivas y creativas desde el conocimiento y práctica de la danza. Para cada uno de los bloques de contenidos distintos se establecen los correspondientes criterios de evaluación que servirán para describir aquello que se quiere valorar, y que el alumnado debe lograr; lo que se pretende conseguir al acabar la etapa. Mediante los estándares de aprendizaje se concreta cada uno de estos criterios de evaluación.

Como resumen de todo lo indicado hasta ahora podríamos concretar que, en el Real Decreto 126/2014 que establece el currículo básico de la Educación Primaria en España, el área de Educación Artística es considerada como asignatura específica, y por tanto dependerá de la regulación de cada Comunidad Autónoma o incluso de los centros educativos su impartición o no. Para la Educación Musical se especifican unos criterios de evaluación y unos estándares de aprendizaje que todo alumno debería alcanzar al acabar su etapa. Por su parte, solo quedan definidos los nombres de los bloques de contenidos, pero sin concretarse los contenidos propiamente dichos, aspecto que sí se especifica en las asignaturas troncales.

La regulación básica actual está planteada, pero ¿qué es lo que ha hecho cada Administración educativa? Analizar cómo se ha precisado todo esto en cada territorio es fundamental para conocer su visión e interés respecto a la Educación Musical, el estilo de enseñanza y el aprendizaje buscado; así como comprobar si un alumno, independientemente del lugar en el que viva, recibe la misma formación musical en España. Para estudiarlo se utilizan varios ejes. Por un lado, si se imparte o no la música y el número de horas reales que tiene en los diferentes lugares; por otro, cuáles son los contenidos, criterios de evaluación y estándares de aprendizaje que la normativa delimita. 


\section{Metodología}

Para la elaboración de este documento se ha realizado un análisis descriptivo y comparativo (Frega, 2001) de los datos obtenidos a través de fuentes documentales, desde una perspectiva cualitativa (Bisquerra, 2000). Este tipo de trabajos "intenta entrar en profundidad en los elementos constitutivos de una situación determinada" (Frega, 1998, p. 109). El tema educativo objeto de estudio es pertinente, ya que "probablemente el estudio de los cambios del currículo y de la enseñanza musical es escaso" (Bresler, 2006, p. 76) y sigue la línea del análisis realizado para áreas como la Educación Física (Méndez, Fernández-Río, Méndez y Prieto, 2015) o competencias como la mediática en la Educación Artística (Ramírez, Renés y Sánchez, 2013).

"La educación se concibe como acción intencionada, global y contextualizada, regida por reglas personales y sociales y no tanto por leyes científicas" (Latorre, Del Rincón y Arnal, 2003, p. 36). Así, y desde esta perspectiva, el propósito principal es interpretar y comprender los fenómenos educativos más que aportar unas explicaciones de tipo causal. De esta manera, se procura desvelar creencias, valores y supuestos que subyacen en la práctica educativa; asimismo, este tipo de trabajo se entiende como un medio permanente de autorreflexión y debería servir para cambiar o mejorar la práctica (Latorre et al., 2003).

Los documentos reflejan y construyen la realidad social y las distintas versiones de los acontecimientos; viéndose hoy en día como los medios a través de los que se expresa el poder social (May, 2001). "Los libros blancos y los documentos legislativos son fuentes importantes para la investigación política" (Blaxter, Hughes y Tight, 2008, p. 185). "Gran parte de la importancia y el interés de los documentos se pone de manifiesto cuando se consideran en relación mutua. Desarrollamos la comprensión de las ideas, cuestiones y políticas tratadas en los documentos mediante un análisis comparativo" (ibídem, p. 225). Latorre (2003, p. 78) considera que el análisis de documentos es una "actividad sistemática y planificada que consiste en examinar (analizar) documentos escritos con el fin de obtener información útil y necesaria para responder a los objetivos de la investigación".

Este trabajo de naturaleza cualitativa intenta responder a tres grandes cuestiones: ¿cuál es el lugar que ocupa la Educación Musical en la actualidad en nuestro país?, ¿cómo está presente la Educación Musical en el currículo actual de Educación Primaria? y ¿existen diferencias educativas significativas dependiendo del lugar en el que se viva?

Para la realización de esta revisión se ha recopilado toda la normativa referente a la enseñanza de la música en Educación Primaria existente en España. Esta normativa incluye tanto la legislación estatal como la de las 17 Comunidades Autónomas, y la que concierne al denominado territorio MEC (Ceuta y Melilla). Se ha procedido a un exhaustivo análisis y comparación de la información manejada, prestando especial atención al objeto de estudio y propósitos planteados. Así pues, se ha realizado una labor de revisión descriptiva e interpretativa de los datos obtenidos (Latorre et al., 2003). La principal limitación que este trabajo puede tener es debida a que la normativa es continuamente cambiante, y su recopilación y análisis se hace en un momento concreto; en el caso que nos ocupa los documentos manejados están actualizados a la fecha de inicio del curso escolar 2016-2017. 


\section{Descripción e interpretación de los datos}

\subsection{Elementos en los diferentes currículos}

Tal y como se indicaba al inicio, la Ley Orgánica actual recoge como elementos integradores del currículo los objetivos, competencias, contenidos, metodología didáctica, estándares y resultados de aprendizaje evaluables y los criterios de evaluación del grado de adquisición de las competencias y del logro de los objetivos. El Real Decreto 126/2014, que establece el currículo básico de la Educación Primaria, solo concreta para el área de Educación Artística, así como para el resto de áreas específicas, una introducción y la relación ordenada por bloques de contenidos de los criterios y estándares de evaluación de la etapa de Primaria. Es por ello que algunas de las Administraciones asumen que el resto de apartados del currículo les compete su especificación, si bien son las menos, como se observa en la Tabla 1. Entre los más desarrollados podemos encontrar Aragón y Canarias, que abordan todos los puntos y concretan la relación de contenidos, criterios y estándares para cada uno de los cursos. Como extremo del menos elaborado encontramos el de Madrid, que sólo incluye una breve introducción y la relación de contenidos, criterios de evaluación y estándares para toda la etapa.

La mayoría de concreciones autonómicas presenta una introducción común a Educación Plástica y Educación Musical y la relación de contenidos, criterios y estándares de evaluación para cada uno de los cursos; siguiendo la línea de concreción curricular iniciada en el Real Decreto. Algunas Comunidades, como Asturias y Valencia, no recogen los estándares de aprendizaje y otras, como Andalucía, Baleares y Cataluña solo lo establecen por ciclos, además de la ya nombrada Madrid que solo indica los de etapa. Los apartados menos abordados son los relacionados con los objetivos y con el desarrollo competencial; la falta de profundización en este último ámbito llama la atención ya que es uno de los aspectos que más se han destacado en educación en las últimas décadas. No debemos olvidar que Ceuta y Melilla están reguladas directamente por el Ministerio de Educación, considerándose las únicas zonas del país gestionadas directamente por las autoridades centrales; respecto al Real Decreto, también en estos territorios se amplían y especifican algunos apartados.

Se debe indicar que la normativa propia para Primaria del País Vasco y Cataluña no se ajusta a las consideraciones que el Real Decreto prescribe en bastantes de sus apartados; entre otros, no respetando los bloques de contenidos establecidos actualmente. Asimismo, Navarra modifica en parte sus enunciados y dimensiones, o Canarias añade un nuevo bloque de contenidos, común para Educación Musical y Educación Plástica denominado Patrimonio cultural $y$ artístico.

Digno también de mención en este apartado resulta constatar cómo el énfasis de las diferentes normas de cada Administración en el ámbito de la evaluación determina, o más bien define, el estilo educativo buscado; ésta aparece por delante de los objetivos, de la metodología, etc. Un análisis minucioso escapa al objetivo concreto de este artículo. 


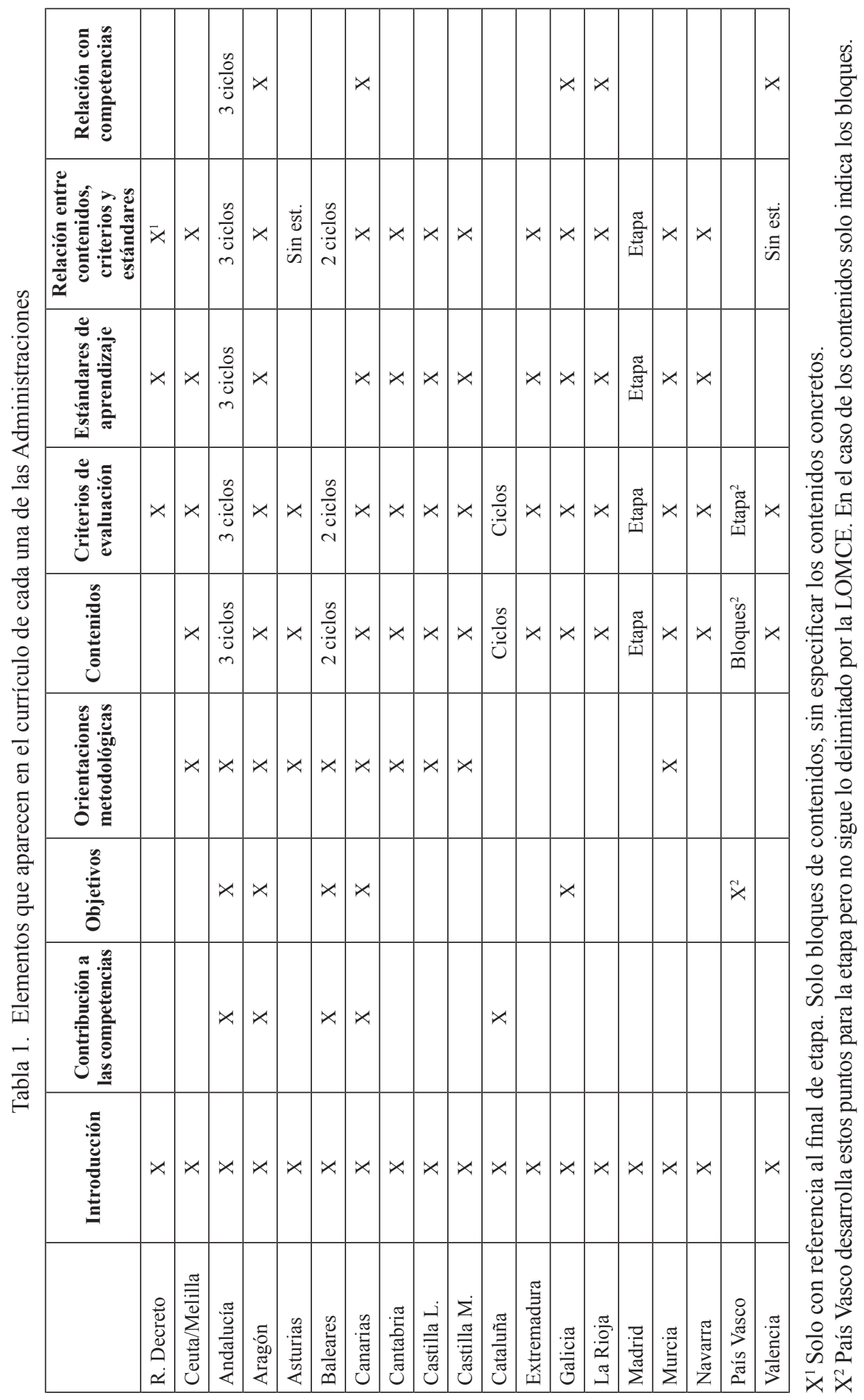




\subsection{Dedicación temporal a la Educación Musical en Primaria}

La actual legislación diferencia entre asignaturas troncales, entre las que recoge Ciencias de la Naturaleza, Ciencias Sociales, Lengua Castellana y Literatura, Matemáticas y Primera Lengua Extranjera; asignaturas específicas obligatorias de cursar, como son Educación Física y Religión, o Valores Sociales y Cívicos; dejando relegada al área de Educación Artística en el grupo de asignaturas específicas en el que al menos hay que cursar una de entre Educación Artística, Segunda Lengua Extranjera, Religión o Valores Sociales y Cívicos, por lo cual deja así abierta la posibilidad de que la Educación Artística no se llegase a trabajar en algunos centros. Pese a que la normativa contempla esta posibilidad de desaparición, la realidad es que todas las Comunidades la ofertan como obligatoria, salvo Madrid que indica que podría llegar a ser sustituida por Segunda Lengua Extranjera si así lo solicitara el Centro y aprobara Inspección.

En general el tiempo mínimo que la norma delimita para el trabajo de Educación Artística es de 2 sesiones por semana y curso. Como en Educación Artística se incluye Educación Plástica y Educación Musical, salvo excepciones concretas en las que se hace mención específica al tiempo a destinar para música, como en el caso de Baleares, Cantabria, Castilla León, Extremadura y La Rioja, se presupone que en la mayoría de los centros españoles esto conlleva que una sesión se destina a Educación Musical y la otra a Educación Plástica. Las sesiones varían entre los 45 minutos y la hora de duración, como norma habitual.

La comunidad que menor tiempo indica como obligatorio para la Educación Artística, además de la anteriormente nombrada Comunidad de Madrid en donde podría llegar a dejarse de impartir en favor de la Segunda Lengua Extranjera, es Murcia, que solo indica para $4^{\circ}, 5^{\circ}$ y $6^{\circ}$ cursos una hora de Educación Artística; con lo cual, está permitiendo que se oferte una única sesión en la que se tenga que trabajar tanto Educación Plástica como Educación Musical. No son excepciones aquellas en las que el mínimo se sitúa en 45 minutos, como son Andalucía, Ceuta y Melilla, Extremadura de $3^{\circ}$ a $6^{\circ}$ cursos, País Vasco al menos en $3^{\circ}, 5^{\circ}$ y $6^{\circ}$, Asturias en $6^{\circ}$ curso, y el caso de Castilla La Mancha si el tiempo se destina a $2^{\mathrm{a}}$ Lengua Extranjera.

Las Comunidades que más tiempo obligatorio ofrecen al área son Canarias con 4 sesiones de 45 minutos en $1^{\circ}, 2^{\circ}$ y $5^{\circ}$ cursos, lo que posibilita tener dos sesiones de música, incluso más. Castilla León marca como mínimo 1 hora, pero estableciendo 2 horas y media mínimas en $1^{\circ}$ y $4^{\circ}$, lo que ofrece la posibilidad a los centros de ofertar 2 sesiones de música en esos cursos. Cataluña indica 2 horas y media, La Rioja 1 hora y media de música en $3^{\circ}$ y $4^{\circ}$. Valencia, si las sesiones son de 45 minutos, concreta que de $1^{\circ}$ a $4^{\circ}$ de Primaria se realizarán 3 sesiones ampliando a 2 horas y cuarto, frente al decrecimiento de la mayoría de las Comunidades.

Aunque lo indicado se refiere a situaciones generales que la normativa establece, se debe considerar que en algunas Comunidades también se deja un tiempo para la denominada Autonomía de Centros con el fin de que cada colegio pueda realizar algún proyecto específico o reforzar aquellas áreas que considere. Pero la práctica habitual es que no suele repercutir en el área artística por la primacía de reforzar las asignaturas troncales, así como para el dominio de una segunda o, incluso, una tercera lengua extranjeras; estas áreas se han convertido en una prioridad en la educación, así como la tecnología, tal y como reza la propia LOMCE en su preámbulo. Si bien, aunque excepcionalmente, encontramos algunos casos en los que sí se ha apostado 
por ofrecer un proyecto musical, que habitualmente es denominado como enseñanzas integradas de música; encontramos esta situación en varios centros de Navarra, Cataluña, Baleares, Aragón y Canarias, entre otros.

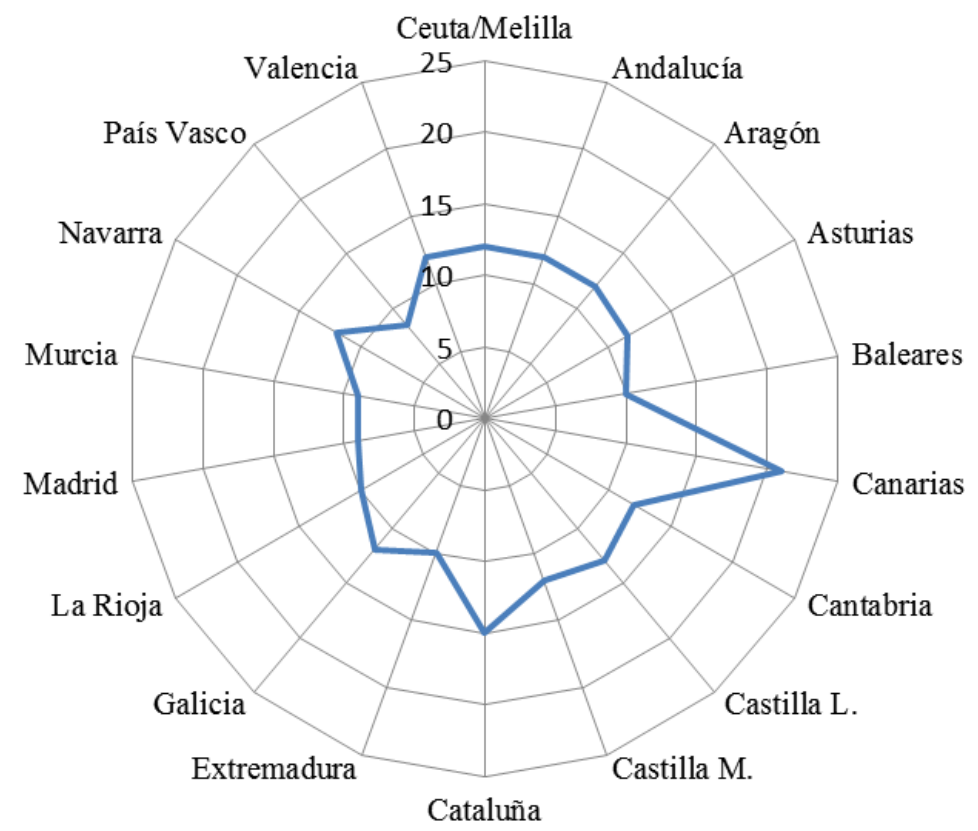

Figura 1. Horas semanales impartidas, de manera general, en Educación Artística en toda la etapa

Asimismo, en algunos casos desde las administraciones educativas de cada Comunidad surgen proyectos para incentivar la Educación Musical. Normalmente dentro del propio horario de clases y a los que se les ofrece también la realización de un concierto extra en un auditorio, como es el caso del Proyecto Cantania en territorios como Cataluña, Madrid, Valladolid, Salamanca, Zaragoza o Sevilla; o el proyecto educativo LÓVA (La Ópera, un Vehículo de Aprendizaje), que comenzó en 2006 en Madrid y en la actualidad su práctica se ha extendido a centros de toda España. También encontramos, entre otros, iniciativas y apoyos para realizar actividades fuera del horario escolar, impartidas por el propio docente de manera gratuita, como por ejemplo el Proyecto de Coros Escolares de Aragón. Hay que resaltar que este tipo de proyectos recaen en la vocación y trabajo extra del profesorado de música que, en casos concretos, acarrea un mayor reconocimiento y aumento de horario en el centro escolar.

\subsection{Aprendizajes de Educación Musical}

Como hemos mostrado anteriormente, todas las Comunidades han desarrollado los contenidos y criterios de evaluación y, en la mayoría de los casos, también los estándares que permiten la concreción de dichos criterios. Basándonos en el estudio de 
estos elementos podemos realizar una aproximación al aprendizaje que el alumnado realizará.

En términos generales podemos decir que un elevado porcentaje de Comunidades han mantenido la misma redacción de los criterios y estándares para $6^{\circ}$ de Educación Primaria que la establecida en el Real Decreto para el final de etapa. Así lo realizan Baleares, Cantabria, Castilla León, Extremadura y Madrid, o con cambios mínimos, como Andalucía, Aragón y Castilla La Mancha. En algunos casos se mantienen los criterios, especificando con mayor detalle los estándares, como en Asturias, Galicia y Murcia y, en menor caso, cambian los criterios, como hacen Canarias y Valencia. Ceuta y Melilla, La Rioja y Navarra modifican tanto los criterios como los estándares. Además encontramos los casos de Cataluña y País Vasco en el que, no acogiéndose a la normativa general, propone unos criterios muy generales.

Un análisis más exhaustivo de la especificación normativa realizada en cada curso nos lleva a destacar que este grado de concreción es significativamente muy diferente. Por un lado encontramos que Madrid y País Vasco no realizan ningún tipo de división por niveles ni especifican los contenidos a trabajar, ni de curso ni de etapa. Baleares establece solo una especificación de $1^{\circ}$ a $3^{\circ}$ y de $4^{\circ}$ a $6^{\circ}$, en el caso de Andalucía y Cataluña, en 3 ciclos; teniendo en cuenta que esta última comunidad no se acoge a la normativa general e indica unos contenidos muy poco concretados, lo que no favorece una secuenciación progresiva de los contenidos a adquirir en los diferentes cursos de la Educación Primaria hasta llegar al final. En el resto sí que se realiza una especificación por cursos, si bien depende mucho de la Comunidad que se hayan graduado todos los criterios o no. En este sentido podemos resaltar que en la mayoría de los casos que se ha hecho esta gradación se ha realizado de los ámbitos musicales tradicionalmente más habituales, como son los relacionados con el conocimiento del lenguaje musical y de la escucha. Sin embargo, otros contenidos como los relacionados con TIC o con la improvisación y creación musical solo son secuenciados en los diferentes cursos en muy pocas comunidades, como Valencia. Esto puede servir de guía y favorecer un proceso de enseñanza-aprendizaje progresivo y funcional para llegar a los niveles de conocimiento de etapa delimitados en cada bloque de contenidos que debería haberse tenido en mayor consideración.

Además, en el caso de que sí se ofrezca una gradación de cada curso, ésta puede ser muy diferente entre una Comunidad y otra. Como ejemplo, podemos citar que las figuras que en Navarra se indican para evaluar en $6^{\circ}$ curso (redondas, blancas, negras, corcheas, y silencios de redondas, blancas y negras) ya son superadas en $3^{\circ}$ en Aragón, curso en el que se incluyen las cuatro semicorcheas así como la corchea y dos semicorcheas combinadas; o en el caso del dominio de notas, en Navarra en $2^{\circ}$ curso deben controlar el do, re, mi, fa, sol, la; cuando en La Rioja, Ceuta y Melilla solo trabajan el sol y el mi. Otro tema es si lo que se ha programado es realista para el tiempo del que se dispone, pero este aspecto se escapa del diseño de este estudio. También deja entrever el estilo de enseñanza-aprendizaje que emana; por ejemplo, en el caso de La Rioja, Ceuta y Melilla que recoge "Identifica y define: figuras, claves (sol y fa), notas, alturas, modo (mayor y menor), textura, matices, carácter", parece poner el énfasis en un trabajo meramente teórico, lo que podría llevarnos al debate de la adecuación de saberes y si éste debe tener un enfoque realmente competencial. 


\section{Discusión y conclusiones}

La finalidad de la Educación Primaria que recoge la normativa educativa actual es facilitar a los alumnos y alumnas los aprendizajes de la expresión y comprensión oral, la lectura, la escritura, el cálculo, la adquisición de nociones básicas de la cultura, y el hábito de convivencia así como los de estudio y trabajo, el sentido artístico, la creatividad y la afectividad, con el fin de garantizar una formación integral que contribuya al pleno desarrollo de la personalidad de los alumnos y alumnas y de prepararlos para cursar con aprovechamiento la Educación Secundaria Obligatoria. Partiendo de esta precisión es mucho lo que la Educación Musical puede ofrecer en la formación buscada en la Educación Primaria, porque desde esta educación específica musical se puede trabajar todos y cada uno de los puntos enumerados. Por ello, en la línea de lo defendido por autores como Aróstegui (2006, 2014a), Cabedo y Díaz (2015) y Ruiz (2015), la música debe y puede contribuir a la consecución de los objetivos del currículo. Pero tal y como se recoge en la normativa vigente, central y autonómica, nuestras autoridades no lo terminan de contemplar.

El hecho de que la normativa estatal la recoja como asignatura específica, abriendo la posibilidad de que las Comunidades o centros educativos puedan llegar a decidir sobre su oferta, menoscaba la importancia que la Educación Musical pueda tener para el desarrollo curricular de Primaria, posibilitando incluso, como resalta Aróstegui (2014b), el cuestionamiento sobre su presencia curricular. La realidad actual es que solo una de todas las Administraciones se ha acogido a esta optatividad, pero sería interesante conocer si las razones reales del resto son la creencia firme de que la Educación Musical contribuye a la formación curricular integral del alumnado. Las horas mínimas de dedicación que cada Comunidad fija y el grado de elaboración y concreción del currículo de esta área también reflejan la importancia reconocida a la misma.

El análisis de los documentos permite observar un mínimo interés por el desarrollo competencial. Asimismo, se constata una mayor tendencia hacia algunos tipos de contenidos de corte más academicista, encontrando en general una falta de gradación en aspectos tales como el trabajo creativo o de integración de las TIC. Por su lado, el énfasis se sitúa en los criterios de evaluación, definidos como los resultados de aprendizaje que responden a los que se pretende conseguir en la asignatura y que deben facilitar el diseño de pruebas estandarizadas y comparables. Esto puede conllevar no tener en cuenta el contexto, el proceso de aprendizaje o centrar la evaluación en el ámbito exclusivamente cuantitativo sin considerar lo cualitativo, es decir, la eficacia de las medidas en el lugar adecuado, con las consecuentes implicaciones que ello acarrea, en la línea de lo apuntado por Aróstegui (2014b) y Parcerisa (2007). A su vez se le une las significativas diferencias de dedicación horaria, de nivel de contenidos, etc. que hacen que las posibles medidas comparativas o evaluaciones entre territorios no puedan realizarse con ningún tipo de rigurosidad. Además, esto puede permitir que un niño que se traslade de una Comunidad a otra encuentre diferencias en ocasiones insalvables o que maestros de diferentes territorios no puedan compartir recursos ni proyectos comunes.

También es necesario plantear la reflexión sobre la posibilidad real de trabajar con la calidad necesaria todo lo que la normativa propone. Para conseguir un desarrollo adecuado y significativo es necesario tiempo y aprovechamiento apropiado de ese tiempo; y en ese sentido el profesorado ocupa un papel determinante. El profesorado 
es el último eslabón en esta cadena de concreción curricular; es el agente mediador del aprendizaje y tiene una perspectiva respecto a la forma óptima para organizarlo de acuerdo con las necesidades del contexto y del alumnado (Alsina, 2010a). Sin embargo, como indica Zaragozà (2009) y se ha mostrado en esta revisión y análisis legislativo, la norma no ayuda pues la carga lectiva mínima suele ser insuficiente para acometer una formación musical completa adecuada.

\section{Repercusiones y prospectiva}

Se hace necesario recordar que "el concepto de competencias supone apostar por una determinada manera de entender la educación y, a la vez, no puede aislarse de otros conceptos ya que educar es una tarea compleja en la que se interrelacionan componentes y aspectos diversos" (Parcerisa, 2007, p. 7). Las competencias "se presentan como la guía de referencia en torno a la cual deben planificarse los procesos de enseñanza-aprendizaje en la educación obligatoria desde todas las áreas" (Zaragozà, 2009, p. 63). La preocupación por formar en competencias responde a la necesidad de que el sistema educativo dé respuestas adecuadas a una realidad personal y social muy distinta de la de apenas hace algunos años, una sociedad en permanente y acelerado cambio (Garagorri, 2007). Por su parte, incluir en los programas formativos contenidos que ayuden a construir una cultura amplia no debería significar la acumulación de contenidos que, en realidad, sólo se justifiquen por la tradición, por la influencia de grupos de presión o por un argumento de autoridad, porque los contenidos, realmente, deberían ser un medio, no un fin en sí mismos (Parcerisa, 2007). La música ofrece un excelente marco competencial, aunque se ha obviado en demasiadas ocasiones por los legisladores educativos (Cabedo y Díaz, 2015).

Asimismo, la máxima del currículo actual es orientar el desarrollo de las capacidades del alumnado; el concepto de educación se ha modificado por la necesidad de coherencia entre lo que se enseña, lo que se aprende y lo que se evalúa (Alsina, 1997). A su vez, vivimos tiempos de cambio de modelo formativo y social, y la Educación Musical y Artística en general, así como el currículo ha de dar respuesta a estos cambios; la presencia de la música en la Educación Primaria debe ser porque fundamentalmente es un medio educativo que contribuye a la consecución de los objetivos del currículo, y si se incluyen determinadas asignaturas ha de ser por esa contribución a los propósitos de todo el sistema escolar (Aróstegui, 2014a; Ruiz, 2015). Aunque la realidad es que asistimos a una grave disfunción del sistema; la escasa carga lectiva de la música condiciona seriamente la consecución de algunos objetivos primordiales del currículo. Para que los objetivos que prescribe la ley tengan una posibilidad real de poder llevarse a término, debe darse la carga lectiva mínima suficiente a las asignaturas, así sí que habrá tiempo para trabajar desde una perspectiva realista (Zaragozà, 2009).

Los criterios de evaluación del actual currículo están definidos en términos de capacidades y no de saberes; es decir, el alumnado, debe ser evaluado en relación a aquello que es capaz de desarrollar y no por lo que sabe (Alsina, 1997). En un actual enfoque por competencias, la evaluación adquiere un papel protagonista que la convierte en el motor del aprendizaje e innovación educativa; todos aquellos aspectos que son objeto de evaluación condicionarán los aprendizajes del alumnado y, por lo tanto, sus conocimientos, habilidades, destrezas y competencias. Toda actividad 
de aprendizaje es evaluable y toda actividad de evaluación debería favorecer, asimismo, el aprendizaje. El enfoque por competencias en general, y su evaluación en particular, ofrece una oportunidad para dar un giro a las modalidades de enseñanzaaprendizaje. Sin embargo, cuando se defiende una estandarización de competencias independientemente de los contextos escolares y se emplea una evaluación cuantitativa que obvia los distintos aspectos cualitativos de la educación, se marca un tipo de evaluación con un enfoque ideológico muy definido (Aróstegui, 2014b). Además, no se puede olvidar que "en educación son muy importantes los procesos y que no siempre conducen directamente ni de modo inexorable a unos resultados definidos teóricamente" (Parcerisa, 2007, p. 8). A su vez, otros procesos deberían cambiar simultáneamente, porque otros muchos contextos oficiales (PISA, por ejemplo) ponen el acento en la evaluación de otras destrezas, encontrándonos, si no es así, en un proceso esquizofrenizante que sólo podrá calificarse de demagogia (Alsina, 2010b).

Ampliando la visión del proceso evaluador, el éxito o el fracaso escolar no se miden solamente a través de los resultados del alumnado, porque la eficacia y la eficiencia de las Administraciones educativas se convierten también en parámetros para medir la calidad educativa. Las Comunidades Autónomas asumen no solamente las competencias normativas de desarrollo normativo estatales, también las plenas competencias ejecutivas y administrativas de la gestión de todo el sistema educativo en su propio territorio. Es evidente que la evaluación es un factor de calidad del sistema educativo, pues contribuye a la mejora de la calidad y la equidad de la educación, orienta las políticas educativas o aumenta la transparencia y la eficacia del sistema, y además, la evaluación, referida a los centros educativos tiene el valor añadido que supone comprobar la eficacia de las medidas en el lugar adecuado y permitir así buscar las soluciones necesarias (Checa, 2012). Para evaluar y comparar se debería contar con las mismas variables, porque si las variables y los elementos son distintos, los resultados serán diferentes. Esas diferencias podrían suponer que un alumno no recibiese la misma formación musical obligatoria, porque ésta dependerá del lugar en el que resida.

Otros aspectos como la promoción de determinadas disciplinas curriculares también definen ideológicamente una postura educativa y conllevan consecuencias a nivel social y cultural. En la actualidad se enfatiza en ciertas áreas o asignaturas consideradas troncales frente a las que no pertenecen a este grupo, entre las que se encuentra la música, ya que "el arte no es visto como una parte valiosa de la educación, pues ni es una disciplina instrumental ni incrementa los ingresos salariales" (Aróstegui, 2006, p. 834). Esta postura educativa, como se ha mostrado en el presente artículo, y apunta Aróstegui (2014b), conlleva enormes consecuencias, llegando incluso a cuestionarse la presencia de la música. Este marco delimitado por la legislación estatal confiere a las Comunidades Autónomas un margen de decisión con respecto a la implementación de la normativa tanto en términos generales como específicos musicales. Este espacio de autonomía afecta a diversos ámbitos de desarrollo y conlleva algunas diferencias, en ocasiones significativas entre los modelos existentes en cada territorio; además, en función del territorio del Estado y del efecto que pueda ejercer la autonomía de cada centro sobre la organización del currículo, las diferencias pueden ser importantes (Alsina, 1997; Zaragozà, 2009).

Como se ha mostrado, las políticas repercuten directamente o indirectamente en la Educación Musical (Hennessy, 2015). Compartimos que "se abren todavía más interrogantes en una sociedad española que continúa sin contar con un sistema educa- 
tivo eficaz, de calidad y consensuado" (Ramírez et al., 2013, p. 683) porque en la actualidad la política educativa no está permitiendo educar ni enseñar para la sociedad de hoy, ni para la sociedad del mañana (Ruiz, 2015). Asimismo, "la configuración curricular de los diferentes niveles del sistema educativo no debe obviar la pregunta acerca de qué educación queremos y el tipo de modelo humano que perseguimos" (Touriñán y Longueira, 2010, p. 176).

A lo largo de este documento han sido contestadas las tres cuestiones planteadas inicialmente. Queda mucha reflexión por hacer. Aquí sólo se ha realizado una aproximación y se han constatado unas circunstancias que se están dando en la actualidad en nuestro país. El análisis debería concretar hacia dónde va la educación en general y la artística en particular; también qué educación queremos en un futuro para nuestros hijos. Porque si no, la Educación Musical está en peligro de desaparecer.

\section{Referencias bibliográficas}

Alsina, P. (1997). El área de educación musical. Propuestas para aplicar en el aula. Barcelona: Graó.

Alsina, P. (2010a). Programar para enseñar musicalmente. En A. Giráldez (Coord.), Didáctica de la Música (pp. 13-34). Barcelona: Graó.

Alsina, P. (2010b). Evaluar en Música. En A. Giráldez (Coord.), Didáctica de la Música (pp. 173-184). Barcelona: Graó.

Aróstegui, J. L. (2006). La formación del profesorado en educación musical ante la convergencia europea en enseñanzas universitarias. Revista de Educación, 341, 829-844.

Aróstegui, J. L. (2014a). Introducción. En J. L. Aróstegui (Ed.), La Música en Educación Primaria. Manual de formación del profesorado (pp. 13-15). Madrid: Dairea ediciones.

Aróstegui, J. L. (2014b). Fundamentos del currículo para la educación musical. En J. L. Aróstegui (Ed.), La Música en Educación Primaria. Manual de formación del profesorado (pp. 19-42). Madrid: Dairea ediciones.

Bisquerra, R. (2000). Métodos de investigación educativa. Guía práctica. Barcelona: CEAC.

Blaxter, L., Hughes, C. y Tight, M. (2008). Cómo se investiga. Barcelona: Graó.

Bresler, L. (2006). Paradigmas cualitativos en la investigación en educación musical. En M. Díaz (Coord.), Introducción a la investigación en Educación Musical (pp. 60-82). Madrid: Enclave Creativa.

Cabedo, A. y Díaz, M. (2015). Arte y música en la educación obligatoria, algo más que un detalle curricular de buen tono. REMIE. Multidisciplinary Journal of Educational Research, 5(3), 268-295. doi:10.17583/remie.2015.1555 Disponible en http://hipatiapress. com/hpjournals/index.php/remie/article/view/1555/1408

Checa, R. (2012). Las políticas educativas de la música a examen. El caso de Andalucía. Eufonía. Didáctica de la Música, 54, 25-35.

Frega, A. L. (1998). La investigación especializada en enseñanzas musicales. Musiker, 10, 101-117.

Frega,A. L. (2001). La investigación en las enseñanzas musicales. Cuadernos Interamericanos de Investigación en Educación Musical, 1(1), 35-47. Disponible en http://www.journals. unam.mx/index.php/cem/article/view/7306

Garagorri, X. (2007). Currículo basado en competencias. Aproximación al estado de la cuestión. Aula de Innovación Educativa, 161, 47-55. 
Hennessy, S. (2015). O jardín o erial: contradicciones entre políticas educativas y prácticas escolares en educación musical. RIEM. Revista Internacional de Educación Musical, 3, 31-40. doi:10.12967/RIEM-2015-3-p031-040 Disponible en http://www. revistaeducacionmusical.org/index.php/rem1/article/view/54

Jefatura del Estado (2006). Ley Orgánica 2/2006, de 3 de mayo, de Educación. Boletín Oficial del Estado, 106, 17.158-17.207. Disponible en http://www.boe.es/boe/dias/2006/05/04/ pdfs/A17158-17207.pdf

Jefatura del Estado (2013). Ley Orgánica 8/2013, de 9 de diciembre, para la mejora de la calidad educativa. Boletín Oficial del Estado, 295, 97.858-97.921. Disponible en http:// www.boe.es/boe/dias/2013/12/10/pdfs/BOE-A-2013-12886.pdf

Latorre, A. (2003). La investigación-acción. Conocer y cambiar la práctica educativa. Barcelona: Graó.

Latorre, A., Del Rincón, D. y Arnal, J. (2003). Bases metodológicas de la investigación educativa. Barcelona: Experiencia.

May, T. (2001). Social Research: Issues, Methods and Process (3 ${ }^{\mathrm{a}}$ Ed.). Buckingham, UK: Open University Press.

Méndez, D., Fernández-Río, J., Méndez, A. y Prieto, J. A. (2015). Análisis de los currículos autonómicos LOMCE de Educación Física en Educación Primaria. Retos, 28, 15-20. Disponible en https://recyt.fecyt.es/index.php/retos/article/view/34822

Ministerio de Educación, Cultura y Deporte (2014). Real Decreto 126/2014, de 28 de febrero, por el que se establece el currículo básico de la Educación Primaria. Boletín Oficial del Estado, 52, 19.349-19.420. Disponible en http://www.boe.es/boe/dias/2014/03/01/pdfs/ BOE-A-2014-2222.pdf

Parcerisa, A. (2007). Las competencias como referentes para la práctica educativa. Eufonía. Didáctica de la Música, 41, 6-16.

Ramírez, A., Renés, P. y Sánchez, J. (2013). Educación artística y competencia mediática en el currículo de Educación Primaria. Historia y Comunicación Social, 18, 673-686. doi:10.5209/rev_HICS.2013.v18.43998 Disponible en http://revistas.ucm.es/index.php/ HICS/article/view/43998

Rodríguez, C. (2014). La proletarización del profesorado en la LOMCE y en las nuevas políticas educativas: de actores a culpables. Revista Interuniversitaria de Formación del Profesorado, 81, 15-29.

Rodríguez, M. y González, E. (2013). Reflexión sobre las competencias básicas y su relación con el currículo. Madrid: Ministerio de Educación, Cultura y Deporte.

Ruiz, C. (2015). Reflexiones sobre políticas educativas de reforma y educación musical. RIEM. Revista Internacional de Educación Musical, 3, 69-73. doi:10.12967/RIEM-20153-p069-073 Disponible en http://www.revistaeducacionmusical.org/index.php/rem1/ article/view/58/28

Touriñán J. M. y Longueira, S. (2010). La música como ámbito de educación. Educación «por» la música y educación «para» la música. Teoría de la Educación, 22(2), 151-181. Disponible en http://revistas.usal.es/index.php/1130-3743/article/view/8300

Zaragozà, J. L. (2009). Didáctica de la música en la educación secundaria. Competencias docentes y aprendizaje. Barcelona: Graó. 